\title{
Diaphragm thickness and stiffness in patients with hyperkyphosis due to osteoporotic vertebral fracture: an ultrasonographic and elastographic study
}

\author{
Osman Ciloglu ${ }^{1 A, B, D, E, F}$, Evren Karaali ${ }^{1 A, B, D}$, Feride Fatma Gorgulu ${ }^{1 B, D, E}$, Timur Ekiz ${ }^{2 A, C, E, F}$ \\ 'University of Health Science Adana City Training and Research Hospital, Turkey \\ ${ }^{2}$ Turkmenbasi Medical Centre, Adana, Turkey
}

\section{Abstract}

Purpose: The objective of this study was to evaluate the thickness and stiffness of the diaphragm, using ultrasound (US) and strain elastography (SE) in patients with hyperkyphosis due to osteoporotic vertebral fracture.

Material and methods: This prospective and case-control study was conducted between October 2019 and December 2019. Diaphragm thickness, SE, and strain ratio values of patients with hyperkyphosis due to osteoporotic vertebral fracture were compared with those of the control group.

Results: There were 42 patients (14 males, 28 females) with a mean age of $81.10 \pm 6.3$ years in the kyphosis group and 36 subjects (11 males, 25 females) with a mean age of $81.00 \pm 5.5$ years in the control group. End-inspirium thickness, change level, and thickening ratio of the diaphragm were significantly higher in the control group $(p<0.001$ for all). Strain ratio values were significantly higher in the kyphosis group, and the rate of hardest colour code was significantly higher in the control group. The diaphragm thickness at end-inspirium and thickening ratio values correlated positively with the forced expiratory volume in the first second $\left(\mathrm{FEV}_{1}, \%\right)$ and forced vital capacity (FVC, \%) values. The strain ratio values correlated inversely with the $\mathrm{FEV}_{1}(\%)$ and FVC (\%) values. The diaphragm thickness at end-inspirium and thickening ratio values correlated inversely with the Cobb values and number of vertebra fractures. A positive correlation was determined between the strain ratio values and the Cobb values and number of vertebra fractures.

Conclusions: Ultrasonography is a promising imaging tool to evaluate and quantify the diaphragm function and stiffness in relevant patients.

Keywords: sonoelastography, musculoskeletal ultrasound, diaphragm, osteoporosis.

\section{Introduction}

Osteoporotic vertebral fractures are commonplace in patients with osteoporosis, particularly in the elderly population, and are most commonly seen in load-bearing areas such as the lower thoracic and upper lumbar vertebrae, resulting in kyphotic posture disorder [1]. The relationship between thoracic kyphosis and respiratory parameters in patients with osteoporosis has been previously studied. In general, due to kyphosis, forced expiratory volume during the first second $\left(\mathrm{FEV}_{1}\right)$ and forced vital capacity (FVC) have been found to be decreased, and a significant association has been determined between kyphosis parameters (e.g. Cobb angle, number of vertebral fractures) and pulmonary function tests (PFTs) [2-4]. The diaphragm is the main respiratory muscle, and its function is associated with the respiratory functions. Although electrodiagnostic tests are the gold standard for the assessment of diaphragm function, they are not easily applicable, due to their invasiveness [5]. In recent years, ultrasound (US) has been used to visualise the diaphragm and assess its

\section{Correspondence address:}

Dr. Osman Ciloglu, University of Health Science Adana City Training and Research Hospital, Mithat Ozhan Bulvari, 01130, Yuregir, Adana, Turkey,

e-mail: osmanciloglu@gmail.com 
function, with several advantages [5,6]. However, to the best of our knowledge, there has been no study evaluating the diaphragm function using B-mode US or sonoelastography in patients with osteoporotic vertebral fractures. Therefore, the objective of this study was to explore the diaphragm thickness and stiffness using US and strain elastography (SE) in patients with hyperkyphosis due to osteoporotic vertebral fracture.

\section{Material and methods}

\section{Study protocol and participants}

This prospective, case-control study was conducted between October 2019 and December 2019. Patients who were diagnosed with osteoporotic vertebral fracture (>20\% reduction in the vertebra height [7] and hyperkyphosis defined as a Cobb angle of $\geq 50^{\circ}[8]$ ) in a tertiary centre constituted the kyphosis group. Participants who met any of the following criteria were excluded: history of smoking, respiratory system diseases (e.g. chronic obstructive pulmonary disease or asthma), neurological diseases (spinal cord injuries, cerebrovascular accidents, neuropathies), any medication usage for respiratory system disorders, cognitive impairment, rheumatic diseases, or any other conditions that may affect the pulmonary system. Vertebra fractures due to trauma or malignancy were also excluded. In addition, a control group was formed of ageand gender-matched healthy subjects without kyphosis.

Physical activity was assessed using the Baecke Questionnaire of Habitual Physical Activity [9]. The severity of kyphosis was measured in all patients using the modified Cobb method [10]. Pulmonary functions of all the participants were evaluated using a spirometer (MasterScreen PFT, Care Fusion, Hoechberg, Germany). FEV ${ }_{1}$ and FVC were measured for each participant [11]. The outcome measures and ultrasonographic values of the two groups were compared.

The current study protocol was approved by the Local Ethics Committee and informed consent was obtained from all participants.

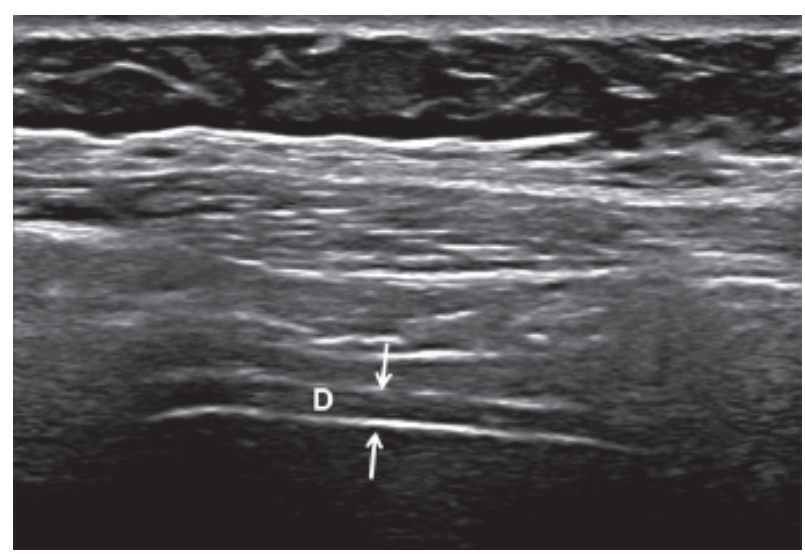

Figure 1. B-mode ultrasound image showing the diaphragm. The diaphragm (D) is visualised between the peritoneum and pleura (thin arrows). The upper and lower white lines correspond to the pleura and peritoneum, respectively

\section{Ultrasound examinations}

Ultrasonographic examinations were administered by the same physician, who was blinded to the patient groups. A high-resolution US Doppler system (Philips ${ }^{\circledR}$ EPIQ 7 Philips Health Care, Bothell, WA, USA) with a high-resolution linear probe (Philips ${ }^{\circledast}$ L5-18) was used for the US examinations. With the patient in a supine position, the transducer was placed over the right chest wall between two costae in the anterior axillary line and was then moved caudally. The diaphragm was visualised as a hypoechoic structure between the peritoneum and pleura (Figure 1). The thickness was measured at the end of expirium and at the end of inspirium $[6,12]$. The difference between the two measurements was defined as the change level ( $\mathrm{mm})$. The thickening ratio (\%) was defined as the percentage (\%) of change level to the thickness at the end of expirium.

At the same level, elastographic images were obtained using SE. SE was graded automatically by the US device as follows: Grade 1 - hardest or hard tissue (red to yellow); Grade 2 - intermediate tissue (green); and Grade 3 - soft tissue (blue) [13]. The mean value of three consecutive measurements in a circular region of interest area at 1-3 mm intervals was taken into consideration (Figure 2). The strain ratio was calculated automatically by the US device.

\section{Statistical analysis}

The data were analysed using the Statistical Package for the Social Sciences software. Normality distribution of the data was checked using histograms and the Shapiro-Wilk test. The descriptive data were given as mean \pm standard deviation or number $(n)$ and percentage (\%). The data of the kyphosis group and control subjects were compared using Student's $t$-test. The $\chi^{2}$ test was used to compare categorical variables between the groups. Pearson's correlation coefficients were used for the correlation analyses. A value of $p<0.05$ was accepted as statistically significant.

The power analysis was performed by GPower 9.1.2 software. The test was chosen as a one-tailed independent sample $t$-test. The effect sizes were determined using the Cobb angle, thickness, spectral reflectance (SR), and FEV and FVC ratios of the kyphosis and control groups. The minimum effect size belonging to the FVC ratio was selected for the analysis. The sample size was determined as 18 patients for each group considering the power as 0.95 and the type-I error as $5 \%$.

\section{Intra-observer agreement}

Ultrasonographic examinations were administered by the same physician, who was blinded to the patient groups. After power analysis, the patient groups were determined and 25 patients were randomly selected from each group. The intraclass correlation coefficient (ICC) was recruited for intra-observer agreement. The ICC values for the end of 


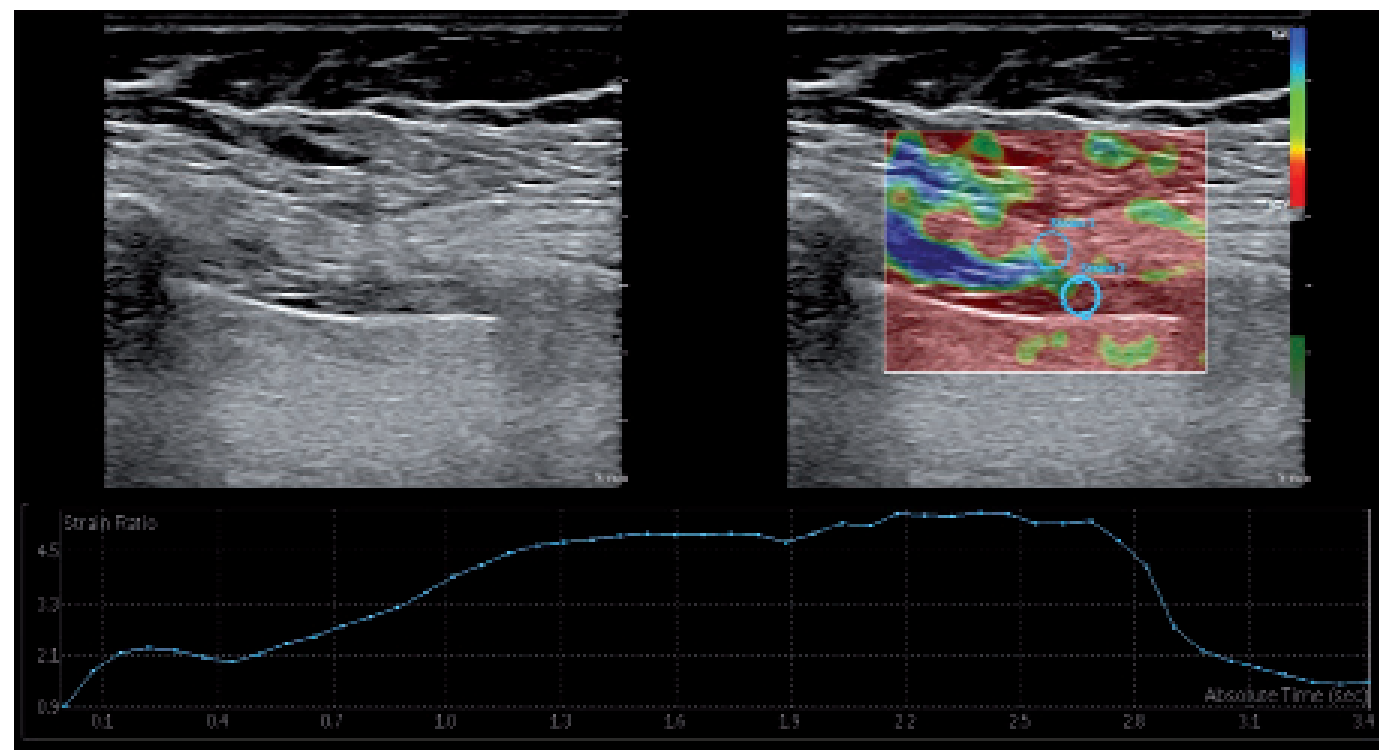

Figure 2. Diaphragm strain ratio measurement using the strain elastography. While measuring the spectral reflectance, the circular region of interest (ROI) was selected at 1-3 mm intervals, and the first ROI was placed in the intercostal muscle as a reference point (strain 1) near the diaphragm (strain 2)

inspirium and expirium of thickness and SR were calculated as $0.816,0.903,0.897$, and 0.926 , respectively. The agreement for colour grades were determined using Kendall's tau test as 0.984 (expirium) and 0.952 (inspirium) $(p<0.001)$.

\section{Results}

A total of 78 subjects were included in the current study, comprising 42 patients ( 14 males, 28 females) with a mean age of $81.10 \pm 6.3$ years (range: 67 to 93 years) in the kyphosis group and 36 subjects ( 11 males, $25 \mathrm{fe}$ males) with a mean age of $81.00 \pm 5.5$ years (range: 69 to 97 years) in the control group. No significant difference was observed between the groups in terms of age, gender, and body mass index ( $p>0.05$ for all).

No significant difference was observed between the groups in respect of the end-expirium thickness $(p=0.553)$. End-inspirium thickness, change level, and thickening ratio values were determined to be statistically significantly higher in the control group ( $p<0.001$ for all). There were significant differences between the groups in terms of strain ratio and SE colour codes. Strain ratio values were significantly higher in the kyphosis group, and the rate of hardest colour code was significantly higher in the control group (Table 1).

The results of the correlation analyses are shown in Table 2. The diaphragm thickness at end-inspirium and thickening ratio values correlated positively with the $\mathrm{FEV}_{1}$ (\%) and FVC (\%) values. The strain ratio values correlated inversely with the $\mathrm{FEV}_{1}$ (\%) and FVC (\%) values. An inverse correlation was determined between the diaphragm thickness at end-inspirium and thickening ratio values and the Cobb values and number of vertebra fractures. The strain ratio values correlated positively with the Cobb values and the number of vertebra fractures. As the Cobb angle or number of vertebra fractures increased, the diaphragm thickness at end-inspirium and thickening ratio decreased, and the strain ratio increased. Correlation analyses between thickening ratio, strain ratio, $\mathrm{FEV}_{1}(\%)$, and FVC (\%) are also illustrated in Figure 3.

\section{Discussion}

The aim of this study was to investigate the thickness and stiffness of the diaphragm using ultrasonography in patients with hyperkyphosis due to osteoporotic vertebral fracture. The study had three primary outcomes. First, the diaphragm thickening ratio was lower in patients with hyperkyphosis compared to that of the control group. Second, the diaphragm stiffness at inspirium decreased in hyperkyphosis patients. Third, PFT parameters such as $\mathrm{FEV}_{1}$ and $\mathrm{FVC}$ decreased in patients with hyperkyphosis compared to those of the control group, and these parameters correlated with the US findings.

US has several advantages in the evaluation of diaphragm morphology and function, such as being easy to apply and non-invasive, the absence of ionising radiation, the provision of dynamic imaging, enabling comparison with the opposite side, and allowing repeated measurements. US also provides information about the diaphragm morphology $[5,14]$. Therefore, US was used in this study. The results showed that diaphragm thickness and the diaphragm thickening rate in inspirium were lower in hyperkyphotic patients. In addition, the US findings were correlated with PFT parameters such as $\mathrm{FEV}_{1}$ and FVC. To the best of our knowledge, this is the first study to have evaluated the diaphragm with US in patients with hyperkyphosis due to osteoporosis, and this is the main strength of the study.

Elastography is a modern sonographic method, evaluating the stiffness of tissues. There are primarily two elastographic methods; SE and shear wave elastography. Compression is produced by the examiner during the SE examination [15]. Hard tissues have a lower strain ratio 
Table 1. Clinical and demographic features

\begin{tabular}{|c|c|c|c|}
\hline Variables & Kyphosis group & Control group & $p$ value \\
\hline Age (years) & $81.10 \pm 6.3$ & $81.00 \pm 5.5$ & 0.945 \\
\hline \multicolumn{4}{|l|}{ Gender } \\
\hline Male & $14(33.3)$ & $11(30.6)$ & \multirow[t]{2}{*}{0.793} \\
\hline Female & $28(66.7)$ & $25(69.4)$ & \\
\hline Body mass index (kg/m²) & $24.68 \pm 2.1$ & $24.56 \pm 1.6$ & 0.783 \\
\hline Baecke Physical Activity Score & $6.79 \pm 0.3$ & $6.78 \pm 0.4$ & 0.880 \\
\hline $\mathrm{COBB}$ & $77.33 \pm 5.4$ & $37.86 \pm 6.7$ & $<0.001$ \\
\hline FEV 1 predicted (It) & $1.77 \pm 0.1$ & $2.14 \pm 0.1$ & $<0.001$ \\
\hline $\mathrm{FEV}_{1}(\%)$ & $90.86 \pm 5.4$ & $105.36 \pm 1.9$ & $<0.001$ \\
\hline FVC predicted (It) & $2.17 \pm 0.1$ & $2.31 \pm 0.1$ & $<0.001$ \\
\hline FVC (\%) & $104.24 \pm 3.1$ & $115.68 \pm 3.1$ & $<0.001$ \\
\hline \multicolumn{4}{|l|}{ Diaphragm thickness } \\
\hline End-expirium (mm) & $1.83 \pm 0.1$ & $1.84 \pm 0.1$ & 0.553 \\
\hline End-inspirium (mm) & $2.97 \pm 0.2$ & $3.58 \pm 0.1$ & $<0.001$ \\
\hline Change level (mm) & $1.13 \pm 0.1$ & $1.74 \pm 0.1$ & $<0.001$ \\
\hline Thickening ratio (\%) & $61.84 \pm 9.6$ & $95.29 \pm 4.3$ & $<0.001$ \\
\hline \multicolumn{4}{|l|}{ Strain ratio } \\
\hline End-expirium & $2.25 \pm 0.1$ & $2.26 \pm 0.1$ & 0.153 \\
\hline End-inspirium & $3.56 \pm 0.1$ & $2.88 \pm 0.1$ & $<0.001$ \\
\hline Change level & $1.31 \pm 0.2$ & $0.61 \pm 0.1$ & $<0.001$ \\
\hline Strain ratio $(\%)$ & $0.58 \pm 0.1$ & $0.27 \pm 0.1$ & $<0.001$ \\
\hline \multicolumn{4}{|c|}{ Strain elastography - end-expirium } \\
\hline Grade 1 (red to yellow) & $6(14.3)$ & $33(91.7)$ & \multirow[t]{3}{*}{$<0.001$} \\
\hline Grade 2 (green) & $34(81.0)$ & $3(8.3)$ & \\
\hline Grade 3 (blue) & $2(4.8)$ & $0(0)$ & \\
\hline \multicolumn{4}{|c|}{ Strain elastography - end-expirium } \\
\hline Grade 1 (red to yellow) & $3(7.1)$ & $27(75.0)$ & \multirow[t]{3}{*}{$<0.001$} \\
\hline Grade 2 (green) & $27(64.3)$ & $9(25.0)$ & \\
\hline Grade 3 (blue) & $12(28.6)$ & $0(0)$ & \\
\hline
\end{tabular}

$\mathrm{FEV}_{1}$ - forced expiratory volume in the first second, FVC - forced vital capacity. The data are shown as mean \pm standard deviation or $n(\%)$. Bold $p$ values denote significance.

Table 2. Correlation analyses

\begin{tabular}{|l|c|c|c|c|c|c|c|c|}
\multicolumn{1}{|c|}{ Variables } & \multicolumn{2}{c|}{ Number of vertebra fracture } & \multicolumn{2}{c|}{ COBB angle } & \multicolumn{2}{c|}{ FEV $_{1}(\%)$} & \multicolumn{2}{c|}{ FVC (\%) } \\
& $r$ & $p$ & $r$ & $p$ & $r$ & $p$ & $r$ & $p$ \\
\hline Thickness end-expirium & 0.086 & 0.453 & 0.052 & 0.651 & -0.104 & 0.365 & -0.131 & 0.252 \\
\hline Thickness end-inspirium & -0.774 & $<0.001$ & -0.931 & $<0.001$ & 0925 & $<0.001$ & 0.787 & $<0.001$ \\
\hline Thickening ratio & -0.772 & $<0.001$ & -0.922 & $<0.001$ & 0.924 & $<0.001$ & 0.795 & $<0.001$ \\
\hline Strain ratio & 0.782 & $<0.001$ & 0.905 & $<0.001$ & -0.929 & $<0.001$ & --0.791 & $<0.001$ \\
\hline
\end{tabular}

and soft tissues have a higher strain ratio [16]. According to the study results, the diaphragm of the control group was coded with harder colours, and strain ratio values were lower in the control subjects. This can be attributed to less contraction of the diaphragms of patients with hyperkyphosis. Similarly, a lower diaphragm thickening ratio in patients with hyperkyphosis was found.
Some mechanisms have been proposed for respiratory function disorders in patients with hyperkyphosis. The limitation of chest mobility in kyphosis is mainly due to the pathological positioning of the ribs and the reduction in the width of the intercostal spaces. The kyphotic posture caused by osteoporosis compression fractures results in structural changes in the thorax. The thorax 

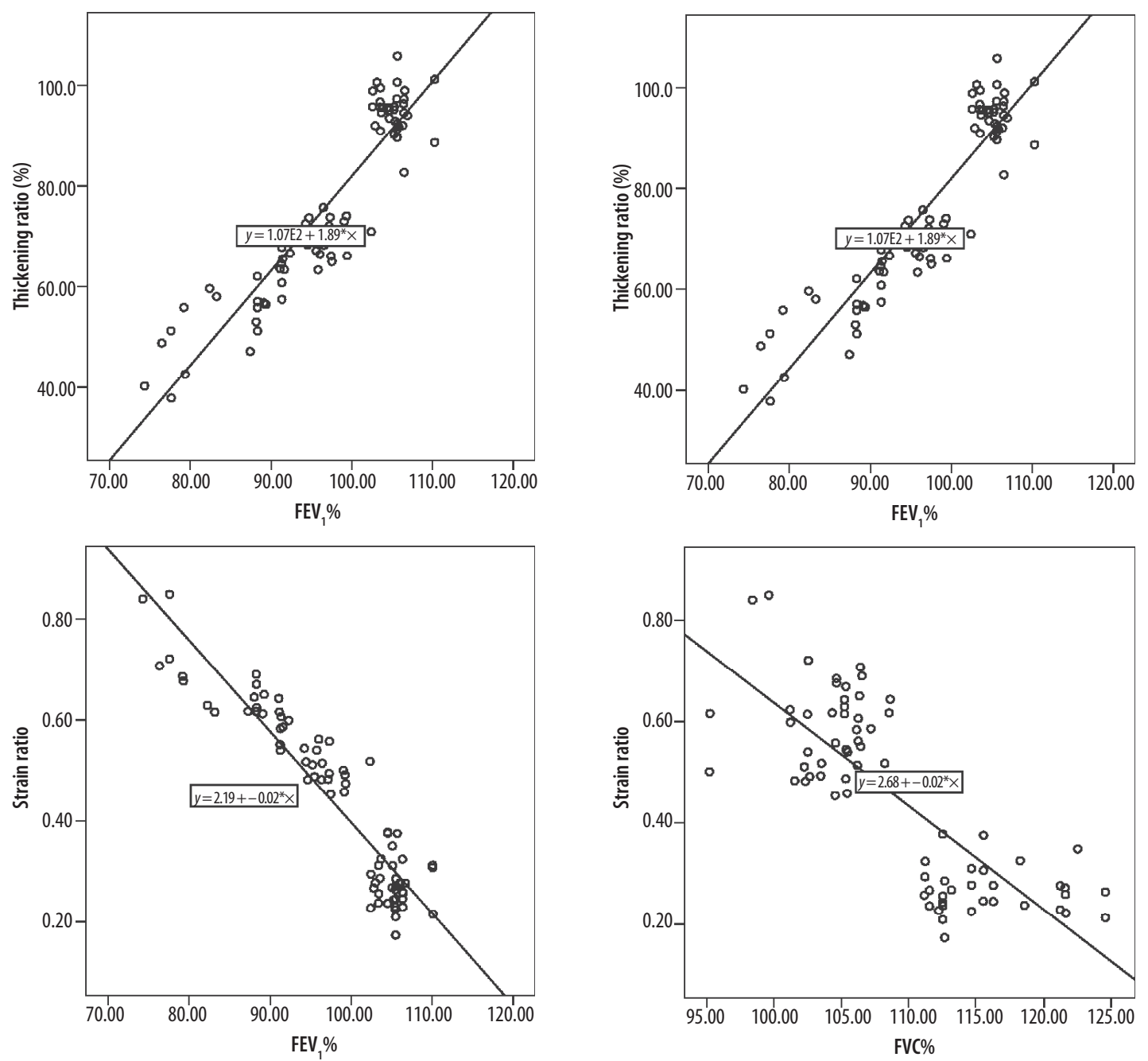

Figure 3. The diaphragm thickening ratio values correlated positively with the forced expiratory volume in the first second $\left(\mathrm{FEV}_{1}, \%\right)(r=0.924, p<0.001)$ and forced vital capacity $(\mathrm{FVC}, \%)(r=0.795, p<0.001)$ values. The strain ratio values correlated inversely with the $\mathrm{FEV}_{1}(\%)(r=-0.929, p<0.001)$ and FVC (\%) values $(r=-0.791, p<0.001)$

moves downwards and approaches the pelvis. In addition, with worsening kyphosis, the intercostal muscles are shortened and their sufficient contractionary powers are reduced. As the volume of the abdominal cavity is reduced due to the displacement of the thorax, the diaphragm is pushed towards the thoracic cavity by the effect of the abdominal organs. While this fact contributes to the reduction of lung volumes, it also creates a mechanical disadvantage for the most important respiratory muscles, including the diaphragm, and reduces the contractibility and effectiveness. Consequently, deformities in the thorax have adverse effects on the individual's respiratory functions, causing mechanical problems as well as volume changes [1,17-19]. In the current study, PFT parameters such as FVC and FEV1 were found to be lower in hyperkyphosis patients compared to those of the control group. PFT parameters were also correlated with values such as the number of vertebra fractures and Cobb angle. In terms of PFT findings, these results are generally consistent with the literature [20].

As for the practical application of sonographic results, diaphragm US measurements can provide overall informa- tion (but cannot replace spirometric tests) about the pulmonary functions of patients with hyperkyphosis. Patients with decreased diaphragm thickening and increased stiffness can be at risk of pulmonary dysfunction. However, we believe that our results should be confirmed with further studies.

\section{Conclusions}

In the light of these first and preliminary results, it can be said that the thickening ratio and stiffness of the diaphragm seem to be decreased in patients with hyperkyphosis due to osteoporotic vertebral fracture. Ultrasonography is a promising imaging tool to evaluate and quantify the diaphragm function and stiffness in relevant patients. Because our study is the first study on this subject, we believe that it should be supported by studies with larger sample sizes.

\section{Conflict of interest}

The authors report no conflict of interest. 


\section{References}

1. Özdemir O, Kutsal Y. The effect of osteoporosis-related thoracic hyperkyphosis on pulmonary function. Osteoporoz Dunyasindan 2010; 16: 61-66.

2. Lombardi I, Oliveira LM, Mayer AF, et al. Evaluation of pulmonary function and quality of life in women with osteoporosis. Osteoporosis Intern 2005; 16: 1247-1253.

3. Ghanbarzadeh M, Mehdipour A. Study and influence of exercise program on respiratory function of adults with kyphosis. Acta Bioeng Biomech 2009; 11: 11-17.

4. Di Bari M, Chiarlone M, Matteuzzi D, et al. Thoracic kyphosis and ventilatory dysfunction in unselected older persons: an epidemiological study in Dicomano, Italy. J Am Geriatr Soc 2004; 52: 909-915.

5. Şahin H, Doğan A, Ekiz T. Ultrasonographic evaluation of the diaphragm thickness in patients with multiple sclerosis. Mult Scler Rel Disord 2019; 36: 101369.

6. Goligher EC, Laghi F, Detsky ME, et al. Measuring diaphragm thickness with ultrasound in mechanically ventilated patients: feasibility, reproducibility and validity. Intensive Care Med 2015; 41: 642-649.

7. Melton L, Egan K, O'Fallon W, et al. Influence of fracture criteria on the outcome of a randomized trial of therapy. Osteoporosis Int 1998; 8: 184-191.

8. Van der Jagt-Willems HC, de Groot MH, van Campen JP, et al. Associations between vertebral fractures, increased thoracic kyphosis, a flexed posture and falls in older adults: a prospective cohort study. BMC Geriatrics 2015; 15: 34

9. Baecke JA, Burema J, Frijters JE. A short questionnaire for the measurement of habitual physical activity in epidemiological studies. Am J Clin Nutr 1982; 36: 936-942.

10. Cobb J. Outline for the study of scoliosis. Instr Course Lect AAOS 1948; 5: 261-275.
11. Miller MR, Hankinson J, Brusasco V, et al. Standardisation of spirometry. Eur Respir J 2005; 26: 319-338.

12. Khurana J, Gartner SC, Naik L, et al. Ultrasound identification of diaphragm by novices using ABCDE technique. Reg Anesth Pain Med 2018; 43: 161-165.

13. Koc AS, Pekoz BC, Donmez Y, et al. Usability of Achilles tendon strain elastography for the diagnosis of coronary artery disease. J Med Ultrason 2019; 46: 343-351.

14. Özçakar L, Kara M, Chang K-V, et al. Nineteen reasons why physiatrists should do musculoskeletal ultrasound: EURO-MUSCULUS/USPRM recommendations. Am J Phys Med Rehabil 2015; 94 : e45-e49.

15. Kantarci F, Ustabasioglu FE, Delil S, et al. Median nerve stiffness measurement by shear wave elastography: a potential sonographic method in the diagnosis of carpal tunnel syndrome. Eur Radiol 2014; 24: 434-440

16. Prado-Costa R, Rebelo J, Monteiro-Barroso J, et al. Ultrasound elastography: compression elastography and shear-wave elastography in the assessment of tendon injury. Insights Imaging 2018; 9: 791-814.

17. De Troyer A, Sampson M, Sigrist S, et al. Action of costal and crural parts of the diaphragm on the rib cage in dog. J Appl Physiol Respir Exerc Physiol 1982; 53: 30-39.

18. Mellin G, Harjula R. Lung function in relation to thoracic spinal mobility and kyphosis. Scand J Rehabil Med 1987; 19: 89-92.

19. Culham EG, Jimenez HA, King CE. Thoracic kyphosis, rib mobility, and lung volumes in normal women and women with osteoporosis. Spine 1994; 19: 1250-1255.

20. Harrison RA, Siminoski K, Vethanayagam D, et al. Osteoporosisrelated kyphosis and impairments in pulmonary function: a systematic review. J Bone Miner Res 2007; 22: 447-457. 